\title{
Role of MicroRNA-155 in Pathogenesis and Prognosis of Preeclampsia pregnancies
}

F.M.Tolba ${ }^{1}$, A.M.Abdelrahman ${ }^{1}$, B.E.Sakr ${ }^{2}$ and S.A.Zaki El Din ${ }^{1}$

${ }^{1}$ Obstetrics and Gynecology Dept., Faculty of Medicine, Benha Univ., Benha, Egypt

${ }^{2}$ Clinical and Chemical Pathology Dept., Faculty of Medicine, Benha Univ., Benha, Egypt

E-Mail:Zaki56@gmail.com

\begin{abstract}
Preeclampsia (PE) is a medical condition characterized by hypertension and proteinuria in pregnant females following 20 weeks of gestation. It is the most common cause of fetal morbidity and mortality, which has no curative therapeutic strategy, except for delivery of the placenta. The association between PE and aberrant microRNA (miRNA) expression in placentas was first reported in 2007 and a number of other similar findings have since been demonstrated.The aim of this study was to assess the level of expression of miR-155 in Egyptian women with preeclampsia and its role in pathogenesis and prognosis of the disease. This study was a case control study included a total of 40 pregnant females; 9 cases with early onset PEdiagnosed $<34$ weeks of gestation whose mean age was 32.4 years, and 21 case of late onset PEdiagnosed $\geq 34$ weeks of gestation whose mean age was 32.2 years admitted to the obstetrics and gynaecology department, Banha University Hospitals, in addition to 10 normotensive pregnant women of matched age as a control group. MIRNA 155 expression level were in PE cases when compared to control groups $(\mathrm{p}<0.001)$. MIRNA 155 expression level showed no significant difference between early onset and late onset PE. miRNA 155 expression showed significantly higher level in PE than normotensive pregnant females with no difference between early and late onset PE.
\end{abstract}

Keywords: MiRNA 155, Expression, Pregnant, preeclampsia, Association.

\section{Introduction}

Preeclampsia(PE) a heterogeneous multisystem disorder defined by the new onset of hypertension and proteinuria after 20 weeks of gestation affects $2-5 \%$ of pregnancies worldwide .Preeclampsia is associated with high risks of iatrogenic preterm delivery, intrauterine growth restriction, placental abruption and perinatal mortality along with maternal morbidity and mortality[1].

Recently, miRNAs have been highlighted as being key regulators capable of influencing various cell processes including differentiation, proliferation, apoptosis, development, as well as angiogenesis and endothelial cell functions, all of which are associated with PE occurrence and manifestation.Furthermore, altered expression of miRNAs has been reportedly linked to multiple pathological diseases such as breast and cervical cancers [2].

MiR-155 has a role in IgA nephropathy [3], Rheumatoid arthritis [4], Spontaneous peritonitis [5],HIV infection[6],Several types of B-cell lymphoma[7]

MicroRNA-155 (miR-155) is a known oncogene and is up regulated in immune cells following exposure to inflammatory stimuli, suggesting an important role in immune regulation. Studies found that miR-155 expression is increased in the placentas of women with $\mathrm{PE}$ at the time of delivery [8].

Overexpression of it contributes to PE by down regulation of cysteine rich protein 61 , decrease in vascular endothelial growth factor, regulates angiotensin2 type 1 receptor expression in umbilical vein endothelial cells from women with severe preeclampsia [7].

\section{Subjects and methods}

The present study was conducted on 40 females 30 patients diagnosed with preeclampsia recruited from gynecology and obstetrics department in Benha university Hospitals, and 10 from normal apparently healthy pregnant females during the periodfrom April 2018 to April 2019 after approval of the local ethical committee of the Gynecology and obstetrics department of Benha University. Informed written consent was obtained from the females after explanation of the study

\section{Study grouping}

The study participiants were divided into 2 main groups

Group I

Included 30 females diagnosed as preeclampsia with the following criteria:Blood pressure $\geq 140 / 90$ $\mathrm{mmHg}$ measured twice least 6 hours apart, Positive proteinuria on urine sticks after 20 weeks of gestation and Normotensive female before pregnancy.

\section{Exclusion criteria}

Women with; Multiple gestations, Fetal congenital malformations or chromosomal abnormalities, Recent infection, Antiphospholipid syndrome, Trauma, Drug or alcohol abuse during pregnancy, Preexisting hypertension, Thrombophilia with history of PE and Receiving anticoagulant or anti-aggregation therapy.

This group is subdivided into 2 subgroups: (a) Early onset PE: group including 9 patients which diagnosed $<34$ weeks of gestation associated with more severe placental and maternal clinical findings(b) Late onset PE group :including 21 patients which was diagnosed $\geq 34$ weeks of gestation with less severe placental and maternal clinical findings.Hemodynamiccharacteristics, frequency of placental lesions are different between early onset preeclampsia and late onset preeclampsia.

\section{Group II}

Included 10 apparently healthy pregnant females 
(control group) with no history of medical illness or use of medication and received routing prenatal care.

\subsection{Methods}

All females included were subjected to the following: Full maternal history taking including: age, gravidity and positive family history. Clinical examination including: Blood pressure (for hypertension) and presence or absence of edema. Laboratory investigations:Venous blood $5 \mathrm{~mL}$ was drawn under complete aseptic conditions and distributed as follows: Whole blood $1 \mathrm{~mL}$ was taken in an EDTA vacutainer and mixed gently. This sample was used to measure complete blood count Whole blood, $4 \mathrm{~mL}$, were taken in plain test tubes without anticoagulant. The separated serum was used for biochemical tests and RNA extraction which used for detection of mir 155 by RT PCR.

\section{Results}

No significant differences were found between groups regarding age ..no significant difference in gravidity and GA. SBP and DBP are higher in early than in late preeclampsia Table (1).

Table (1) Comparison of pregnant demographic, anthropometric, obstetric and clinical data between early and late PE cases.

\begin{tabular}{|c|c|c|c|c|c|c|}
\hline \multirow[b]{2}{*}{ Maternal age (years) } & \multirow[b]{2}{*}{ mean $\pm \mathrm{SD}$} & \multicolumn{2}{|c|}{$\begin{array}{c}\text { Early } \\
N=9\end{array}$} & \multicolumn{2}{|c|}{$\begin{array}{c}\text { Late } \\
\mathrm{N}=\mathbf{2 1}\end{array}$} & \multirow{2}{*}{$\begin{array}{c}\mathbf{p} \\
0.778\end{array}$} \\
\hline & & 32.4 & 6.1 & 32.2 & 6.2 & \\
\hline Gravidity & Median (range) & 0 & $0-2$ & 0 & $0-2$ & 0.071 \\
\hline GA (weeks) & mean $\pm \mathrm{SD}$ & 28.4 & 5.2 & 29.8 & 5.4 & 0.426 \\
\hline SBP (mmHg) & mean $\pm \mathrm{SD}$ & 181.3 & 26.9 & 150.5 & 8.8 & $<0.001$ \\
\hline DBP (mmHg) & mean $\pm \mathrm{SD}$ & 111.6 & 8.9 & 104.7 & 11.2 & 0.114 \\
\hline
\end{tabular}

No significant differences were found in miRNA155 expression level between early and late preeclampsia Table (2).

Table (2) Comparison of miRNA155 expression level and fold between early and late PE cases.

\begin{tabular}{lccccccc}
\hline & \multicolumn{3}{c}{$\begin{array}{c}\text { Early } \\
\text { N=9 }\end{array}$} & \multicolumn{2}{c}{$\begin{array}{c}\text { Late } \\
\text { N=11 }\end{array}$} & \multirow{2}{*}{ p } \\
\cline { 2 - 8 } RU155 & Median & \multicolumn{2}{c}{ range } & Median & \multicolumn{2}{c}{ range } & \\
\cline { 2 - 7 } & 160327.1 & 36711.9 & 516021.3 & 126314.7 & 34267.3 & 856017.4 & 0.447 \\
\hline
\end{tabular}

\section{Discussion}

Preeclampsia (PE) is one of the leading causes of maternal and neonatal morbidity and mortality. Preeclampsia is associated with aberrant expression of several MicroRNAs which function as gene regulators. The present study aims to determine the expression of MicroRNA-155 in Preeclampsia, and to detect the association of MicroRNA-155 levels with early and late preeclampsia

In our study, miRNA 155 expression level were significantly higher in PE cases when compared to control groups $(\mathrm{p}<0.001)$.

[9] reported that miRNA-155 level was significantly increased in $\mathrm{PE}$ pregnancies compared to healthy pregnancies $(\mathrm{P}=.020)$. They confirmed that miR-155 was upregulated in the PE pregnancies compared with healthy pregnancies.

[10] demonstrated a significant increase $260 \%$ in the levels of miR-155 in the severe PE placentas, as compared with the controls.

[8] found that levels of miRNA-155 expression were significantly increased in PE placentas and serum, compared to the normal group $(\mathrm{P}<.05)$.

[11] demonstrated that the PE group had high significant increases in relative expressions of
MicroRNA-155 with about 2 folds increases more than the control group.

Various studies as [12] stated that the level of miRNA-155 expression was upregulated in preeclampsia pregnant females.

In contrast, [13] found no statistically significant difference in miRNA expression levels between total cases of PE, compared to the control group $(p=0.239)$

In the present study, no significant differences were found in miRNA155 fold between early and late PE cases.

In accordance, [13] found no statistically significant difference in miRNA expression levels between early PE and late PE either when compared to each other or compared to the control group.

[15] reported that miR-155 was noted as a PE marker, and as determinant of its severity. MiR-155 was demonstrated to interfere with trophoblast migration and hinder placental angiogenesis, thus promoting PE pathogenesis.

In contrast, [11] reported that MicroRNA-155 could not be used as a prognostic biomarker for severity of Preeclampsia.

Logistic regression analysis was conducted for prediction of PE development. Higher miRNA 155 
expression level was considered as an independent predictor for PE development.

A recent study [14] confirmed that miRNA-155 was up-regulated in women with PE compared to the control group.

\section{Conclusion}

The findings of study demonstrated that miRNA 155 expression showed significantly higher level in PE than normotensive pregnant females and no significant difference between early and late preeclampsia.

\section{Funding}

There was no funding for this work.

\section{Declaration of competing interest} interest.

The authors declare that there are no conflicts of

\section{References}

[1] H. Zeisler, E.Liurba, F. Chantraine, F. Vastish ,Predictive value of the sflt-1:PIGF ratio in women with suspected preeclampsia.NEngi J Med , Vol.(374), PP. 13-22, 2016.

[2] X. Dai , Y.Cai ,Down regulation of microRNA let $7 \mathrm{~d}$ inhibits the proliferation and invasion of trophoblst cells in preeclampsia. Journal of cellular biochemistry, Vol.119(1), PP.1141 1151, 2017.

[3] Qin, Tang,Yang , "Potential role of circulating microRNAs as a biomarker for unexplained recurrent spontaneous abortion," Fertility and Sterility, Vol. 105(5), PP. 1247-1254, 2016.

[4] L. Su, A .Huang, H. Jia, Y. Liu ,Role of microRNA-155 in Rheumatoid arthritis. Internal journal of Rheumatoid diseases ;Vol.(10),PP.11111756,2017

[5] P. Lutz, M. Haimid, A .Pohlmann, J. Lehmann ,Micro RNA-155 is up regulated in ascites in patients with spontaneous bacterial Peritonitis. Scientific Report, Vol.(10),PP.10-38,2017.

[6] C. Jin, L. Cheng, S. Hoxtermann, T. Xie ,MicroRNA-155 is abiomarker of t-cell activation and immune dysfunction in HIV-1-infected patients. HIV medicine, Vol.18(5),PP.354$362,2017$.

[7] K .Bounds, V .Chiasson, L. Pan, S. Gupta , MicroRNAs: New players in the pathobiology of preeclampsia. Front cardiovasc Med, Vol.4, PP.60, 2017.

[8] X. Yang, J. Zhang , Y.Ding ,Association of microRNA-155, interleukin17A (Baltimore);Vol.96(18),PP.6509,2017.

[9] Gan, Liu,Wei , MiR-210 and miR-155 as potential diagnostic markers for preeclampsiaregnancies. Medicine, Vol. 96(28), PP.18-8, 2017.

[10]X. Li, C. Li, X. Dong, MicroRNA-155 inhibits migration of trophoblast cells and contributes to the pathogenesis of severe preeclampsia by regulating endothelial nitric oxide synthase. Mol Med Rep, Vol.10, PP.550-4, 2014.

[11]H. M. G.Youssef, E. S. Marei, Association of MicroRNA-210 and MicroRNA-155 with severity of preeclampsia. Pregnancy Hypertension, Vol. 17, PP.49-53,2019.

[12]D.A. Enquobahrie, D.F. Abetew, T.K. Sorensen, D. Willoughby, K. Chidambaram , M.A. Williams, Placental microRNA expression in pregnancies complicated by preeclampsia. Am J Obstet Gynecol, Vol. 204, PP.12-21, 2011.

[13] S .Demirer, M. Hocaoglu, A. Turgut, A. Karateke, E.Komurcu - Bayrak ,Expression profiles of candidate microRNAs in the peripheral blood leukocytes of patients with early-and late-onset preeclampsia versus normal pregnancies. Pregnancy Hypertension, Vol.8(5), PP.14-5 , 2019.

[14] D. S.Jairajpuri, Z. H.Malalla, N.Mahmood, W. Y. Almawi, Circulating microRNA expression as predictor of preeclampsia and its severity. Gene, Vol.627, PP.543-548, 2017.

[15]D. su, Micro RNA-155 contributes to preeclampsia by down-regulating CYR61. Am J ObstetGynecol, Vol.202, PP.466-7, 2010. 